Supporting Information for Publication

\title{
A Specific Set of Heterogeneous Native Interactions Yields Efficient Knotting in Protein Folding
}

\author{
João NC Especial ${ }^{1,2} \&$ Patrícia FN Faísca ${ }^{1,2 *}$
}

${ }^{1}$ Departamento de Física and ${ }^{2}$ BioISI - Biosystems and Integrative Sciences Institute, Faculdade de Ciências, Universidade de Lisboa, Campo Grande, Ed. C8, Lisboa, Portugal

Corresponding author:

Patrícia FN Faísca (Email: pffaisca@fc.ul.pt)

This file includes:

Supporting Tables S1:S2

Supporting Figures S1:S4 


\begin{tabular}{|c|c|c|c|c|c|c|c|c|c|c|c|c|}
\hline \multirow{2}{*}{\multicolumn{2}{|c|}{$\begin{array}{l}\text { Native } \\
\text { Contact }\end{array}$}} & \multicolumn{4}{|c|}{$\begin{array}{l}\text { Contact probability } \\
\text { profile classification } \\
(\text { Sigmoidal }=1)\end{array}$} & \multicolumn{4}{|c|}{$\begin{array}{l}\text { The } 16 \text { native contacts } \\
\text { present in I intermediate }\end{array}$} & \multicolumn{3}{|c|}{$\begin{array}{l}\text { Native contacts } \\
\text { that precede knot }\end{array}$} \\
\hline & & Go & S1 & S2 & S3 & Go & S1 & S2 & S3 & Go & $\mathrm{S} 2$ & S3 \\
\hline 2 & 11 & 1 & 0 & 0 & 0 & 1 & 0 & 0 & 0 & & 0 & 0 \\
\hline 2 & 15 & 0 & 0 & 0 & 0 & 0 & 0 & 0 & 0 & & 0 & 0 \\
\hline 2 & 39 & 0 & 0 & 0 & 0 & & & & & & & \\
\hline 2 & 41 & 0 & 0 & 1 & 0 & & & & & & & \\
\hline 3 & 18 & 1 & 0 & 0 & 0 & 1 & 0 & 0 & 0 & 1 & & \\
\hline 3 & 20 & 1 & 1 & 0 & 0 & 1 & 1 & 0 & 0 & 1 & 0 & 0 \\
\hline 3 & 24 & 1 & 1 & 1 & 0 & 1 & 1 & 1 & 0 & 1 & 1 & 0 \\
\hline 3 & 30 & 1 & 0 & 1 & 0 & 1 & 0 & 1 & 0 & & 1 & \\
\hline 4 & 23 & 1 & 1 & 1 & 0 & 1 & 1 & 1 & 0 & 1 & 1 & \\
\hline 4 & 31 & 1 & 0 & 0 & 0 & & & & & 1 & & \\
\hline 4 & 33 & 1 & 1 & 0 & 1 & & & & & 1 & & 1 \\
\hline 5 & 20 & 1 & 1 & 1 & 1 & 1 & 1 & 1 & 1 & 1 & 1 & 1 \\
\hline 5 & 22 & 1 & 1 & 1 & 0 & 1 & 1 & 1 & 0 & 1 & 1 & \\
\hline 5 & 34 & 1 & 1 & 0 & 1 & & & & & & & \\
\hline 6 & 35 & 0 & 1 & 0 & 1 & & & & & & & \\
\hline 7 & 22 & 0 & 0 & 0 & 0 & & & & & & & \\
\hline 8 & 21 & 1 & 0 & 1 & 1 & & & & & 1 & 1 & 1 \\
\hline 9 & 38 & 0 & 1 & 0 & 0 & & & & & & & 0 \\
\hline 10 & 21 & 1 & 1 & 0 & 0 & 1 & 1 & 0 & 0 & 1 & 0 & 0 \\
\hline 10 & 39 & 0 & 0 & 0 & 0 & & & & & & & 0 \\
\hline 11 & 24 & 1 & 0 & 0 & 0 & 1 & 0 & 0 & 0 & & 0 & 0 \\
\hline 12 & 15 & 0 & 1 & 0 & 0 & 0 & 1 & 0 & 0 & & 0 & 0 \\
\hline 12 & 25 & 0 & 0 & 1 & 0 & 0 & 0 & 1 & 0 & & & \\
\hline 14 & 29 & 0 & 0 & 0 & 0 & & & & & & & \\
\hline 15 & 30 & 0 & 1 & 1 & 0 & 0 & 1 & 1 & 0 & & 1 & \\
\hline 16 & 41 & 0 & 0 & 0 & 0 & & & & & & & \\
\hline 17 & 30 & 1 & 1 & 0 & 0 & & & & & & & \\
\hline 17 & 32 & 0 & 1 & 0 & 0 & & & & & & & \\
\hline 18 & 33 & 1 & 1 & 0 & 1 & & & & & & & \\
\hline 18 & 41 & 0 & 0 & 0 & 0 & & & & & & & \\
\hline 19 & 34 & 1 & 1 & 1 & 0 & & & & & & & 0 \\
\hline 19 & 36 & 0 & 0 & 0 & 0 & & & & & & & \\
\hline 19 & 40 & 0 & 0 & 0 & 0 & & & & & & & \\
\hline 20 & 39 & 0 & 0 & 0 & 0 & & & & & & & 0 \\
\hline 21 & 24 & 0 & 1 & 1 & 1 & 0 & 1 & 1 & 1 & 0 & 1 & 1 \\
\hline 23 & 26 & 0 & 0 & 0 & 0 & & & & & & & \\
\hline 25 & 30 & 0 & 1 & 1 & 0 & 0 & 1 & 1 & 0 & & 1 & \\
\hline 26 & 31 & 0 & 0 & 0 & 0 & & & & & & & \\
\hline 28 & 31 & 0 & 0 & 0 & 0 & & & & & & & \\
\hline 37 & 40 & 0 & 0 & 0 & 0 & & & & & & & \\
\hline \multirow{5}{*}{\multicolumn{2}{|c|}{$\begin{array}{l}\text { Sigmoidal } \\
\text { Non-sigmoidal } \\
\text { Total } \\
\text { Sigmoidal } \\
\text { Non-sigmoidal }\end{array}$}} & 17 & 18 & 12 & 7 & 10 & 10 & 9 & 2 & 10 & 9 & 4 \\
\hline & & 23 & 22 & 28 & 33 & 6 & 6 & 7 & 14 & 1 & 6 & 11 \\
\hline & & 40 & 40 & 40 & 40 & 16 & 16 & 16 & 16 & 11 & 15 & 15 \\
\hline & & $43 \%$ & $45 \%$ & $30 \%$ & $18 \%$ & $63 \%$ & $63 \%$ & $56 \%$ & $13 \%$ & $91 \%$ & $60 \%$ & $27 \%$ \\
\hline & & $57 \%$ & $55 \%$ & $70 \%$ & $82 \%$ & $37 \%$ & $37 \%$ & $44 \%$ & $87 \%$ & $9 \%$ & $40 \%$ & $73 \%$ \\
\hline
\end{tabular}

Table S1. Classification of native contact probability profiles into sigmoidal- and nonsigmoidal-shaped. The function $[1+\tanh (a Q+b)] / 2$ was fitted to each contact probability profile, through adjusting parameters $a$ and $b$ using the non-linear least squares MarquardtLevenberg algorithm, initialized with $a=1$ and $b=-0.5$. Contact probability profiles were classified as sigmoidal-shaped if and only if the final sum of squares of residuals amounted to less than 0.1 , all residuals being weighted equally (unit weight). A contact was said to precede the knot if the $Q_{0.8}$ of its contact probability profile was smaller than the $Q_{0.8}$ of the $p_{k n o t}$ profile. 


\begin{tabular}{|c|c|c|c|c|c|c|c|c|c|}
\hline \multicolumn{9}{|c|}{ Native } & \multicolumn{5}{c|}{ Non-native } \\
\hline $\begin{array}{c}\text { p in } \\
\text { knotted }\end{array}$ & Contact & Energy & $\begin{array}{c}\mathrm{p} \text { in } \\
\text { unknotted }\end{array}$ & $\Delta \mathrm{p}$ & $\begin{array}{c}\mathrm{p} \text { in } \\
\text { knotted }\end{array}$ & Contact & Energy & $\begin{array}{c}\mathrm{p} \text { in } \\
\text { unknotted }\end{array}$ & $\Delta \mathrm{p}$ \\
\hline 0,999915 & $(11-24)$ & $-0,97$ & 0,944065 & 0,055850 & 0,719075 & $(4-29)$ & $-0,41$ & 0,124147 & 0,594928 \\
\hline 0,999915 & $(3-30)$ & $-0,97$ & 0,773533 & 0,226382 & 0,692490 & $(26-29)$ & $-0,41$ & 0,334243 & 0,358247 \\
\hline 0,999915 & $(3-24)$ & $-0,97$ & 0,956344 & 0,043571 & 0,588943 & $(7-20)$ & $-0,42$ & 0,384720 & 0,204223 \\
\hline 0,999915 & $(2-11)$ & $-0,76$ & 0,083220 & 0,916695 & 0,559309 & $(1-10)$ & $-0,29$ & 0,008186 & 0,551123 \\
\hline 0,999831 & $(25-30)$ & $-0,97$ & 0,998636 & 0,001195 & 0,559224 & $(1-20)$ & $-0,14$ & 0,283765 & 0,275459 \\
\hline 0,999746 & $(5-20)$ & $-0,42$ & 0,954980 & 0,044766 & 0,479384 & $(14-31)$ & $-0,29$ & 0,147340 & 0,332044 \\
\hline 0,999746 & $(3-20)$ & $-0,38$ & 0,964529 & 0,035217 & 0,366269 & $(2-17)$ & $-0,39$ & 0,664393 & $-0,298124$ \\
\hline 0,999661 & $(2-15)$ & $-0,76$ & 0,210095 & 0,789566 & 0,365168 & $(1-18)$ & $-0,29$ & 0,105048 & 0,260120 \\
\hline 0,999577 & $(10-21)$ & $-0,39$ & 0,952251 & 0,047326 & 0,347896 & $(1-16)$ & $-0,26$ & 0,181446 & 0,166450 \\
\hline 0,999492 & $(21-24)$ & $-0,45$ & 0,983629 & 0,015863 & 0,332656 & $(13-26)$ & $-0,25$ & 0,206003 & 0,126653 \\
\hline 0,999069 & $(15-30)$ & $-0,97$ & 0,948158 & 0,050911 & 0,219033 & $(16-33)$ & $-0,26$ & 0,115962 & 0,103071 \\
\hline 0,998730 & $(12-25)$ & $-0,76$ & 0,862210 & 0,136520 & 0,210482 & $(18-39)$ & $-0,76$ & 0,073670 & 0,136812 \\
\hline 0,998391 & $(12-15)$ & $-0,76$ & 0,994543 & 0,003848 & 0,201592 & $(34-37)$ & $-0,20$ & 0,234652 & $-0,033060$ \\
\hline 0,992803 & $(5-22)$ & $-0,42$ & 0,933151 & 0,059652 & 0,172043 & $(32-35)$ & $-0,11$ & 0,170532 & 0,001511 \\
\hline 0,992549 & $(4-23)$ & $-0,41$ & 0,944065 & 0,048484 & 0,149945 & $(16-39)$ & 0,11 & 0,170532 & $-0,020587$ \\
\hline 0,981627 & $(3-18)$ & $-0,76$ & 0,945430 & 0,036197 & 0,146135 & $(35-38)$ & $-0,07$ & 0,175989 & $-0,029854$ \\
\hline 0,837694 & $(8-21)$ & $-0,29$ & 0,844475 & $-0,006781$ & 0,140462 & $(31-34)$ & $-0,20$ & 0,070941 & 0,069521 \\
\hline 0,790534 & $(28-31)$ & $-0,41$ & 0,821282 & $-0,030748$ & 0,137414 & $(30-39)$ & $-0,97$ & 0,353342 & $-0,215928$ \\
\hline 0,633308 & $(17-30)$ & $-0,45$ & 0,081855 & 0,551453 & 0,130980 & $(28-37)$ & $-0,41$ & 0,167804 & $-0,036824$ \\
\hline 0,597071 & $(23-26)$ & $-0,41$ & 0,627558 & $-0,030487$ & 0,127170 & $(38-41)$ & $-0,09$ & 0,079127 & 0,048043 \\
\hline
\end{tabular}

Table S2. The top 20 most probable contacts formed in the knotted ensemble of the intermediate state of S1. Native contacts (left), and non-native contacts (right) ordered by decreasing probability of formation; their corresponding intramolecular contact energy, their probability of formation in the unknotted ensemble and the difference between the two probabilities, $\Delta \mathrm{p}=(\mathrm{p}$ in knotted $)-(\mathrm{p}$ in unknotted $)$, are also reported. We highlight in orange native contacts that may not be relevant for knotting and in green non-native ones that may play a role in knotting (see also Fig. S4). 

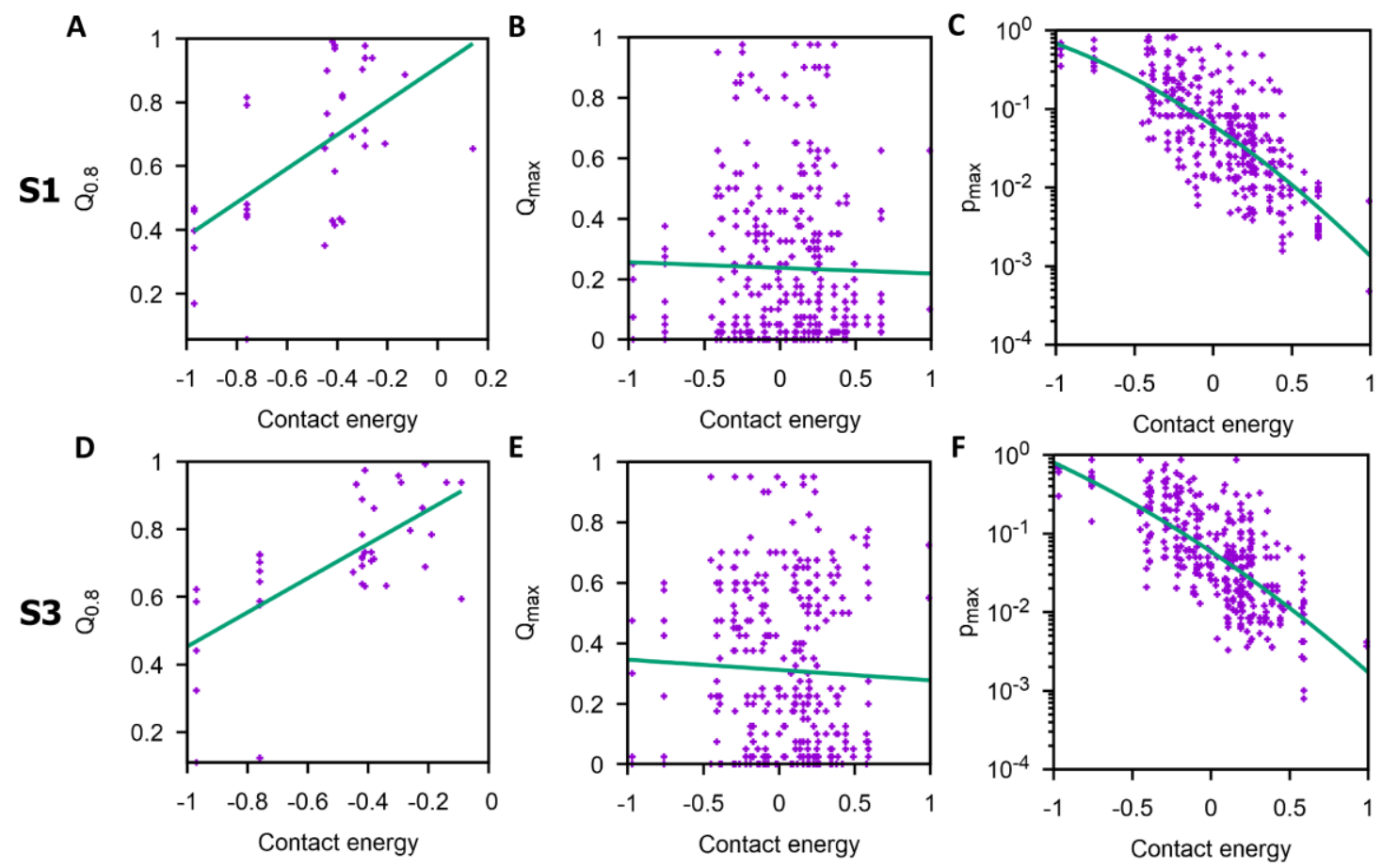

Fig. S1. Relationship between $Q_{0.8}, Q_{\max }, \mathrm{p}_{\max }$ and the intramolecular contact energies. Scatterplot of the (contact energy, $Q_{0.8}$ ) pairs for the 40 native contacts of S1 (A) and of S3 (D). A clear trend exists, the more energetically stable native contacts tending to form earlier. Scatterplot of the (contact energy, $Q_{\max }$ ) pairs for the 340 non-native contacts of S1 (B) and of S3 (E). No trend exists. Scatterplot of the (contact energy, $\mathrm{p}_{\max }$ ) pairs for the 340 non-native contacts of S1 (C) and of S3 (F). A clear trend is visible, the more energetically stable nonnative contacts achieving higher probabilities along the folding pathway. To establish the trends, for each of the S1 and S3 sequences, three models are fitted using least squares: For native contacts, (1) $Q_{0.8}$ as a linear function of contact energy and for non-native contacts, (2) $Q_{\max }$ as a linear function of contact energy and (3) $\mathrm{p}_{\max }$ as a quadratic function of contact energy. To infer whether the dependent variable significantly depends on the independent variable a two-tailed t-test hypothesis test is performed for linear and quadratic coefficients. The null hypothesis is that the mean of the coefficient's sampling distribution is zero and from its acceptance no dependence would be inferred. The values of $t$ reported in the main text are the test's t ratio. The reported p-values are the smallest significance levels at which the null hypothesis would be rejected and, hence, dependence would be inferred. All calculations were performed using the R statistical analysis package. 


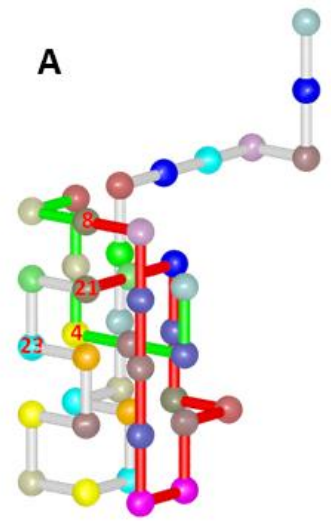

B

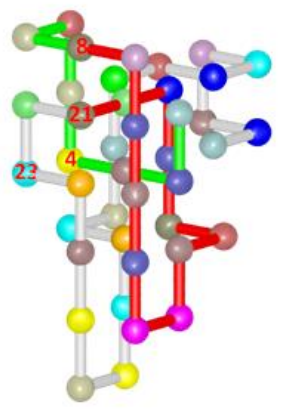

Fig. S2. Representative conformations of the largest (4607 conformations) (A), and smallest (1709 conformations) knotted clusters (B), of the ss 16 intermediate state. 

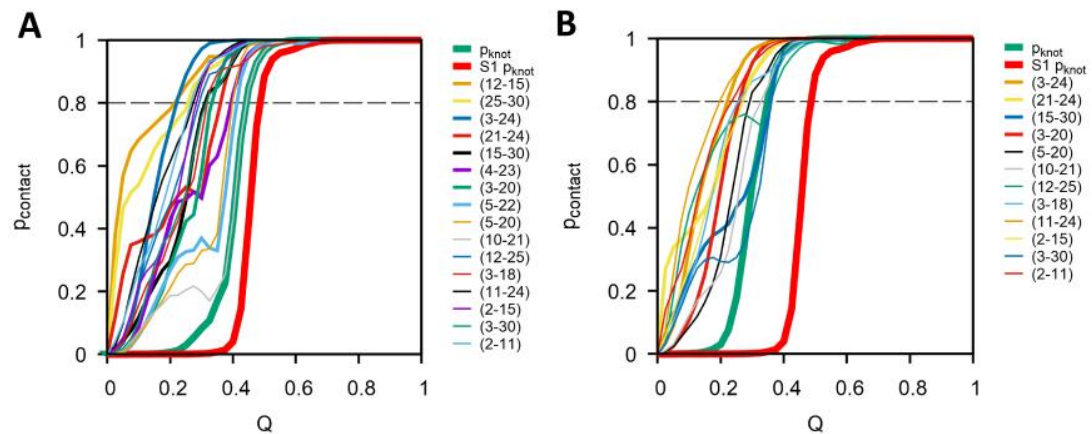

Fig. S3. Contact probability profiles. Contact probability profiles for the 16 native contacts that precede knot formation in the sequence $\mathrm{S} 1$ obtained for the $\mathrm{ss} 16^{\circ}$ potential (A), and contact probability profiles of the 12 native contacts obtained for the $s s 12^{0}$ potential (B). 
A

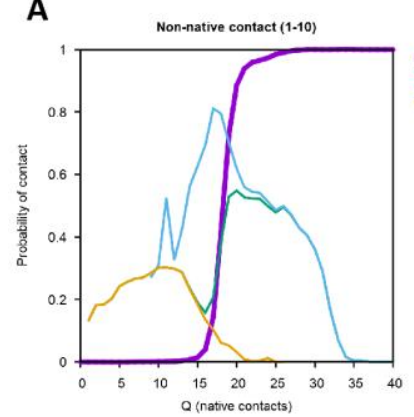

B

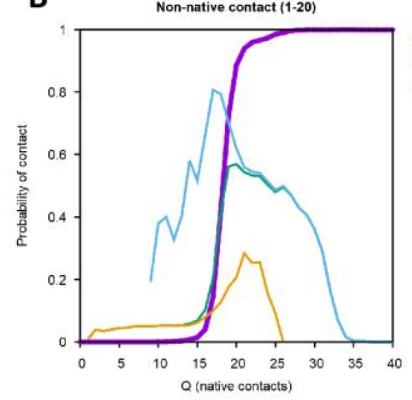

c

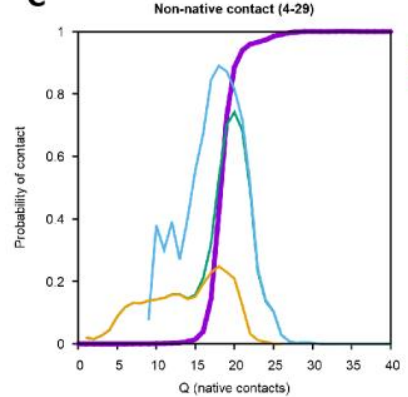

D

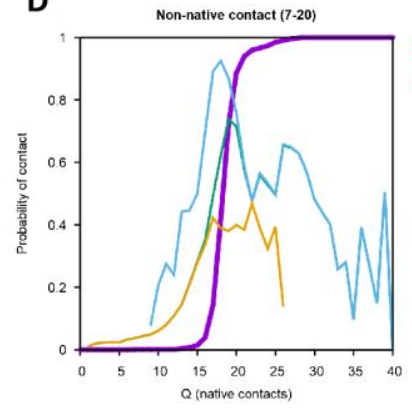

E

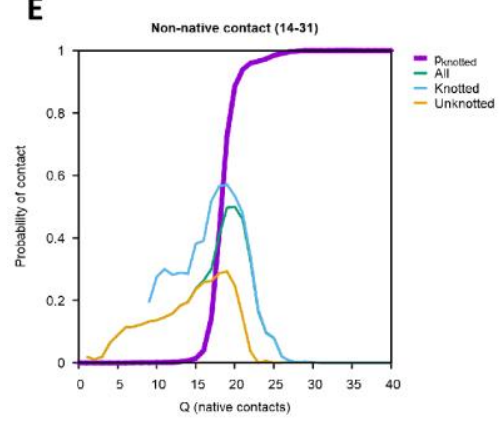

$\mathbf{F}$
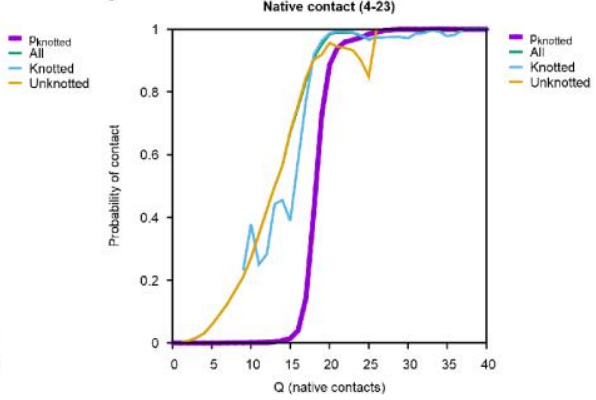

G
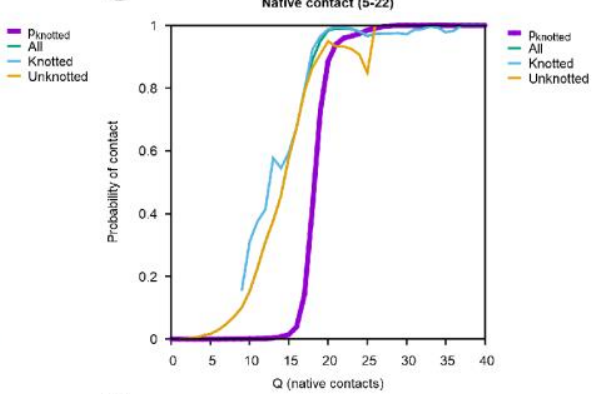

H
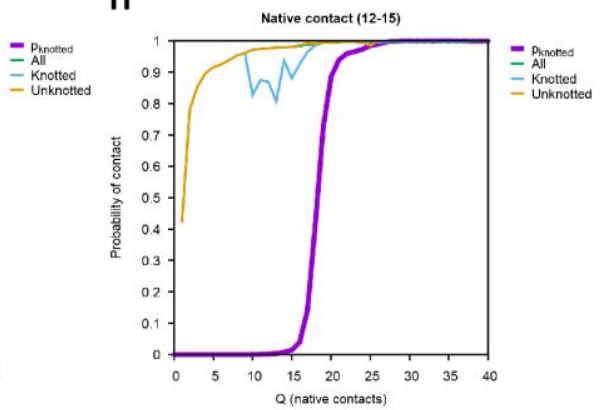

I
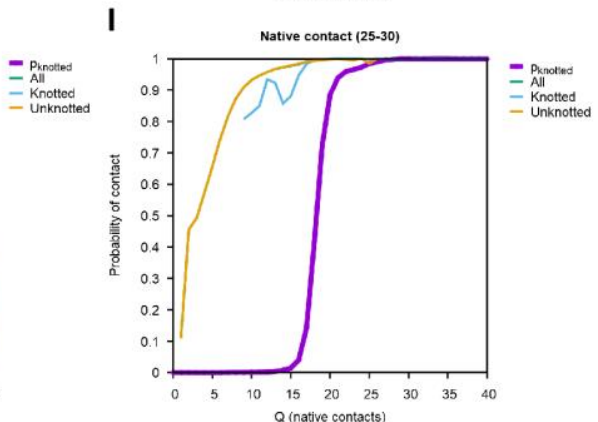

Fig. S4. Notable contact probability profiles. Contact probability profiles for the 5 non-native contacts that most facilitate knot formation in sequence S1 (A-E), and contact probability profiles of the 4 native contacts removed from potentials $s s 16^{+2}$ and $s s 16^{0}$ to obtain the native component of potentials ss $12^{+5}$ and $\mathrm{ss} 12^{0}$ (F-I). 